\title{
CEREUS
}

DOI:10.18605/2175-7275/cereus.v9n1p.146-162

\section{CUSTOS E INTERNAÇÕES HOSPITALARES POR PNEUMONIA EM CRIANÇAS VACINADAS PELA PCV10 EM REGIÃO METROPOLITANA DO TOCANTINS}

\author{
ARANTES, Rhenan Vilela ${ }^{1}$ \\ SILVA, João Paulo Malheiros Costa ${ }^{1}$ \\ VASCONCELOS, Lucas Viníssios Silva ${ }^{1}$ \\ ALMEIDA, Paulo Victor Dias ${ }^{1}$ \\ GLEHN, Gustavo José Von ${ }^{2}$ \\ BESSA, Nelita Gonçalves Faria de ${ }^{3}$
}

\section{RESUMO}

Objetivo: Analisar os gastos e o número de internações por pneumonia de crianças entre 0 e 4 anos na RIDE de GurupiTO em períodos pré e pós vacinal. Metodologia: Foi feito estudo retrospectivo-analítico utilizando dados do Departamento de Informática do Sistema Único de Saúde (DATASUS) de crianças residentes da região metropolitana RIDE de Gurupi -TO (17020), período de 2004 a 2015. A associação entre as variáveis, número de internações, gastos

\footnotetext{
${ }^{1}$ Acadêmicos do curso de Medicina, Centro Universitário UnirG, Gurupi-TO. E-mail: rhenanarantes16@gmail.com; dr.joaopaulomalheiros@gmail.com; lucasvinissios2@hotmail.com; paulo-victor-almeida@hotmail.com.

2Doutor em Anestesiologia, Docente do curso de Medicina, Semiologia, Centrc Universitário UnirG, Gurupi-TO. E-mail: profgustavoglehn@yahoo.com.br. ${ }^{3}$ Doutora em Biologia e Ecologia Tropical, Docente do curso de Medicina, Pesquisa em Saúde, Centro Universitário UnirG, Gurupi-TO E-mail:eduambiental@unirg.edu.br
}

v. 9, n. 1, jan/abr. 2017 UnirG, Gurupi, TO, Brasil 
com internações e cobertura vacinal foi analisada usando a correlação de Pearson. Nos períodos pré-vacinal e pósvacinal foram analisados 02 grupos etários e por sexo: (A) menores de um ano e (B) de 1 a 4 anos, separados por sexo masculino (1) e feminino (2); para a análise entre os períodos antes e depois, subdivididos em $\mathrm{A}$ e $\mathrm{B}$, com número de internações, gastos hospitalares e nascidos vivos, aplicou-se o teste T e Mann-Whitney. Resultados e Discussão: Nasceram 250.996 crianças em Tocantins entre 2005 e 2014, com média anual de 25.099,6, sendo 28.845 e 2.884,5, respectivamente, na RIDE Gurupi. O gasto total foi de $\mathrm{R} \$ 2.175 .903,12$ com internações no período de 12 anos distribuídos entre o grupo A $(R \$ 764.210,55)$ e $B$ $(R \$ 1.411 .692,57)$. Houve redução do gasto público $(-13,21 \%)$ com saúde e número de internação (-36\%) após a PCV10. Foram internadas 3.846 crianças entre 0 e 4 anos e custo médio de $\mathrm{R} \$ 565,75$ por internação. Conclusão: Os custos com internação por pneumonia em crianças foram significativamente menores no grupo feminino, embora reduzidos também para o sexo masculino e grupo etário. Houve uma redução significativa nos números dessas internações no período pós-vacinal independente do grupo etário e gênero.

Palavras-chave: Internação. Infância. Pneumonia. Custos Hospitalares. Epidemiologia.

\section{COSTS AND HOSPITALIZATIONS BY PNEUMONIA IN CHILDREN VACCINATED BY PCV10 IN METROPOLITAN REGION OF TOCANTINS}

\section{ABSTRACT}

Objective: to analyze hospitalization costs for children aged 0 to 4 years in GURUPI-RIDE in pre and post-vaccination 
periods. Methodology: A retrospective-analytical study was carried out, using data from the Department of Informatics of the Unified Health System (DATASUS) in children living in the metropolitan region of Gurupi -TO (17020) from 2006 to 2015. In the pre- (A) under 1 year and (B) 1 to 4 years, separated by male (1) and female (2). The ASSISTAT program version 7.7 beta was used. For the analysis between periods before and after, subdivided into $A$ and $B$, between admissions, hospital expenses and live births, the $T$ and Mann-Whitney tests were applied. Results and Discussion: A total of 3,846 children were admitted between 0 and 4 years of age. A total of 250,996 children were born in Tocantins with an annual average of $25,099.6$, with $(n=28,845) 9.99 \%$ in Gurupi RIDE, with an annual average of 2,884.5 in the period from 2005 to 2014. Total expenditure was $2,175,903.12(\mathrm{~N}=764.210,55)$ and $B(n=1,411,692.57)$, being indicative of the reduction of public health expenditure. Conclusion: The hospitalization costs were significantly lower in the female group, although also reduced for males and the age group. There was a significant reduction in the numbers of hospitalizations in the post-vaccination period with PCV10, regardless of age group and gender.

Key Words: Hospitalization. Childhood. Pneumonia. Hospital Costs. 


\section{INTRODUÇÃO}

As doenças infecciosas respiratórias agudas ainda têm elevada incidência na população infantil e sua gravidade potencial tem justificado os avanços por meio da prevenção vacinal. A pneumonia bacteriana, septicemia e meningite são responsáveis, anualmente, por aproximadamente 800 mil mortes no mundo entre crianças menores de cinco anos de idade, tendo como um dos principais agentes etiológicos 0 Streptococcus pneumoniae, acometendo em 41,9\% dos casos nesta faixa etária (MENEZES et al., 2011). Há evidências quanto à necessidade de adoção de medidas preventivas contra esta doença invasiva, podendo inferir que a vacina oferece cerca de $60 \%$ de proteção (GOMES, 2001).

O Brasil foi um dos primeiros países no mundo a implantar no Serviço Público de Saúde a vacina pneumocócica conjugada 10 valente (PCV10), incluída no calendário de vacinação da criança por meio do Programa Nacional de Imunização do Ministério da Saúde em 2010, disponibilizada em três doses, aos dois, quatro e seis meses de idade, e com reforço aos 12 meses (Calendário básico de vacinação), proporcionando um maior controle da doença pneumocócica. Estudos realizados em diversos países, incluindo o Brasil, demonstram uma tendência de maior acometimento de meningite pneumocócica em crianças do sexo masculino (AZEVEDO, TOSCANO e BIERRENBACH, 2013). Grando et al. (2015) verificaram redução de $50 \%$ e $69 \%$, respectivamente, de morbidade e mortalidade de meningite pneumocócica após 02 anos da PCV10, sendo este fato associado à introdução da vacina. Nos Estados Unidos foi registrada uma redução de $64 \%$ na incidência da meningite pneumocócica em menores de dois anos após cinco anos de uso da PCV7, cuja cobertura é para uma menor variação de sorotipos.

Segundo boletim da Organização Mundial de Saúde (OMS) de 2008, o Brasil encontra-se entre os 15 países com maior incidência de pneumonia, apresentando cerca de 1,8 milhões de casos/ano, o que equivale a aproximadamente $\quad 0,11$ episódios/criança/ano (YOSHIOKA et al., 2011). Estudos publicados pelo 
Ministério da Saúde concluem que a PCV10 tem sido eficaz no impacto de internações por pneumonia em crianças menores que dois anos, onde em 16 municípios houve registro somente $29,8 \%$ de internações por pneumonia diante de um total de 722.351 internações, além de evidências pré e pós-vacinal quanto a diminuição nas internações em Natal ($61,7 \%)$, Curitiba $(-44,6 \%)$, Vitória ($40,6 \%)$, Belo Horizonte (-29,5\%), Distrito Federal (-4,7\%), Florianópolis ($21,2 \%)$, Teresina $(-16,8 \%)$, e Fortaleza $(-9,0 \%)$ (BRASIL, 2014).

Portanto já existem evidências que sugerem a efetividade

\section{METODOLOGIA}

Trata-se de um estudo retrospectivo-analítico, com população de crianças residentes no estado de Tocantins, inter-relacionadas a região metropolitana-RIDE de Gurupi-TO (17020), que foram internadas por pneumonia, conforme lista de morbidade CID-10 e gastos hospitalares no período de 2004 a 2015, além dos grupos de nascidos vivos no período de 2005 a 2014 .

A coleta de dados foi realizada na base de dados do Departamento de dessa vacina, contudo, são ainda desconhecidos tais dados em regiões metropolitanas do Estado do Tocantins. Também não existem inferências quanto a possível economia de gastos públicos, especialmente na região Norte do País e Estado do Tocantins, decorrentes da implementação dessa vacinação em crianças de 0 a 5 anos de idade. Diante destes fatos, o objetivo do presente estudo foi analisar os gastos e o número de internações por pneumonia de crianças entre 0 e 4 anos na RIDE de Gurupi-TO, entre 2006 a 2015. Ademais, a relação entre gastos e nascidos vivos também foi considerada.

Informática do Sistema Único de Saúde (DATASUS), utilizando os grupos epidemiológicos através da morbidade hospitalar do SUS para dados relativos ao número de internações e gastos gerais com saúde, grupo de nascimentos ocorridos, contados segundo o local de residência da mãe e assistência à saúde e grupo imunizados por PCV10.

Foram analisados dados de crianças entre 0 a 4 anos, divididos em dois grupos etários: (A) menores de 
um ano e (B) de 1 a 4 anos, separados por sexo masculino (1) e feminino (2), divididos em dois períodos: pré-vacinal de 2004 a 2009 e outro pós-vacinal de 2010 a 2015. Os grupos de nascidos vivos na região foram considerados para comparar se no decorrer dos anos houve diferença nos números de nascidos no período de 2005 a 2014 (dados cadastrados no SUS). Para validação do período pré e pós vacinal por PCV10 foram utilizados dados da cobertura vacinal por imunização de pneumococo, registrados a partir de 2010 pelo SUS. O programa EXCEL 2013 foi utilizado para análise primária de: média, incidência, porcentagem e regressão linear simples. Foi feita análise de correlação de Pearson, onde

\section{RESULTADOS}

No período do estudo (2004 a 2015) foram internadas 3.846 crianças entre 0 e 4 anos, grupo $A(n=1.278$; $33,23 \%)$ e grupo $B(n=2.568 ; 66,77 \%)$ por pneumonia na região metropolitana de Gurupi- TO, sendo: 55,09\% $(n=2.119)$ do sexo masculino, distribuído em crianças em idade lactente (grupo $\mathrm{A} 1 ; \mathrm{n}=736$ ) e préescolar (grupo B1; $n=1.383$ ); e 44,91\% $(n=1.727)$ do sexo feminino (grupo A2, se verificou o grau de associação entre as variáveis, número de internações, gastos com internações e cobertura vacinal durante o período pré e pósvacinal, usando para tal análise o software SAS (Statistical Analysis System, versão 9.3). Para comparar os grupos entre idade, sexo e nascimento foi usado o programa ASSISTAT, versão 7.7 beta. Os testes de normalidade utilizados foram o Lilliefors e o Shapiro-Wilk. Para a análise entre os períodos pré-vacinal e pós-vacinal, subdivididos em $A$ e $B$, entre internações, gastos hospitalares e nascidos vivos aplicou-se o teste $T$ e Mann-Whitney dependendo da distribuição de dados.

$n=542$; grupo $B 2 ; n=1.185$ ), conforme Figura 1.

A análise levando em conta o período e o número de internação pelos grupos etários e por sexo foi, de maneira geral, satisfatória mediante o coeficiente de determinação $\left(R^{2}=0,5293\right)$ apresentado a partir do modelo escolhido para tal avaliação (Figura 2). 
O sistema de saúde teve gasto total de $\mathrm{R} \$ 2.175 .903,12$ com internações em 12 anos analisados, sendo grupo A $(R \$=764.210,55)$ e $B$ $(\mathrm{R} \$=1.411 .692,57)$. Os gastos masculinos $(R \$=1.198 .175,82)$ foram para o grupo $A 1(R \$=442.319,6)$ e $B 1$ ( $R \$=755.856,22)$, sendo os gastos femininos $(R \$=977.727,30)$ menores para o grupo $A 2(R \$=321.890,95)$ e $B 2$ $(\mathrm{R} \$=655.836,35) \quad$ (Figura 3). $\quad \mathrm{De}$ maneira geral, o modelo de análise foi capaz de explicar os dados coletados $\left(R^{2}=0,0572\right)$, considerando a totalidade dos grupos e respectivos valores no período analisado (Figura 4).

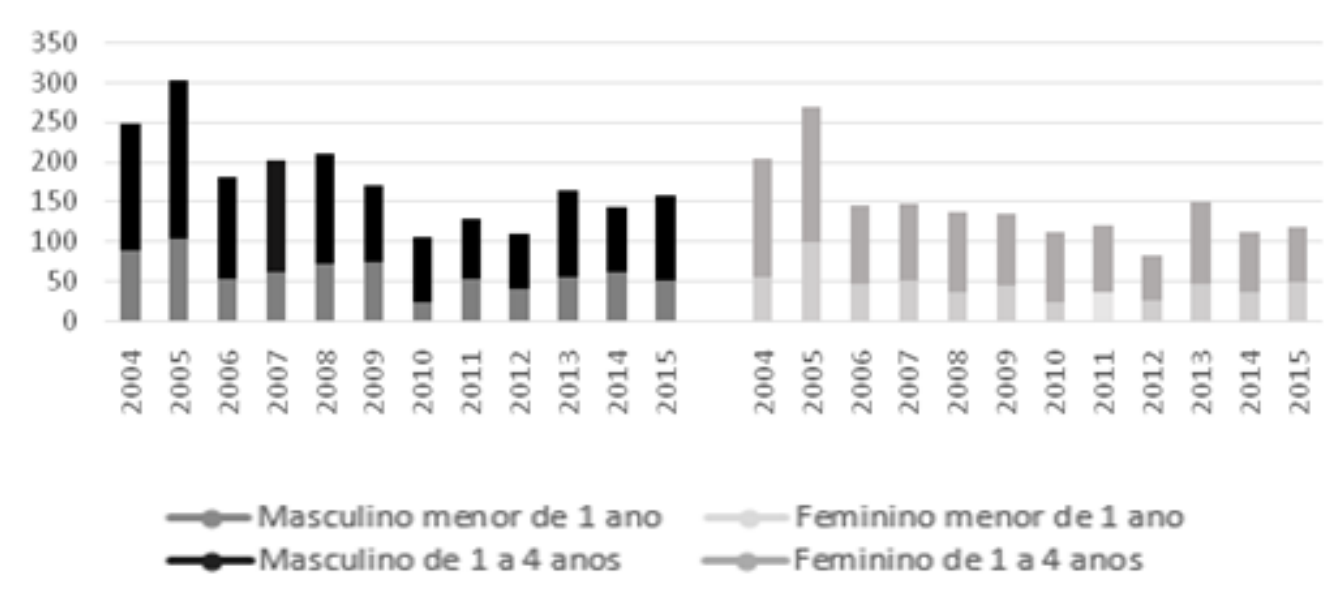

Figura 1. Número de Internações hospitalares de crianças com pneumonia no período de 2004 a 2015 por grupos etários e sexo, RIDE-Gurupi-TO.

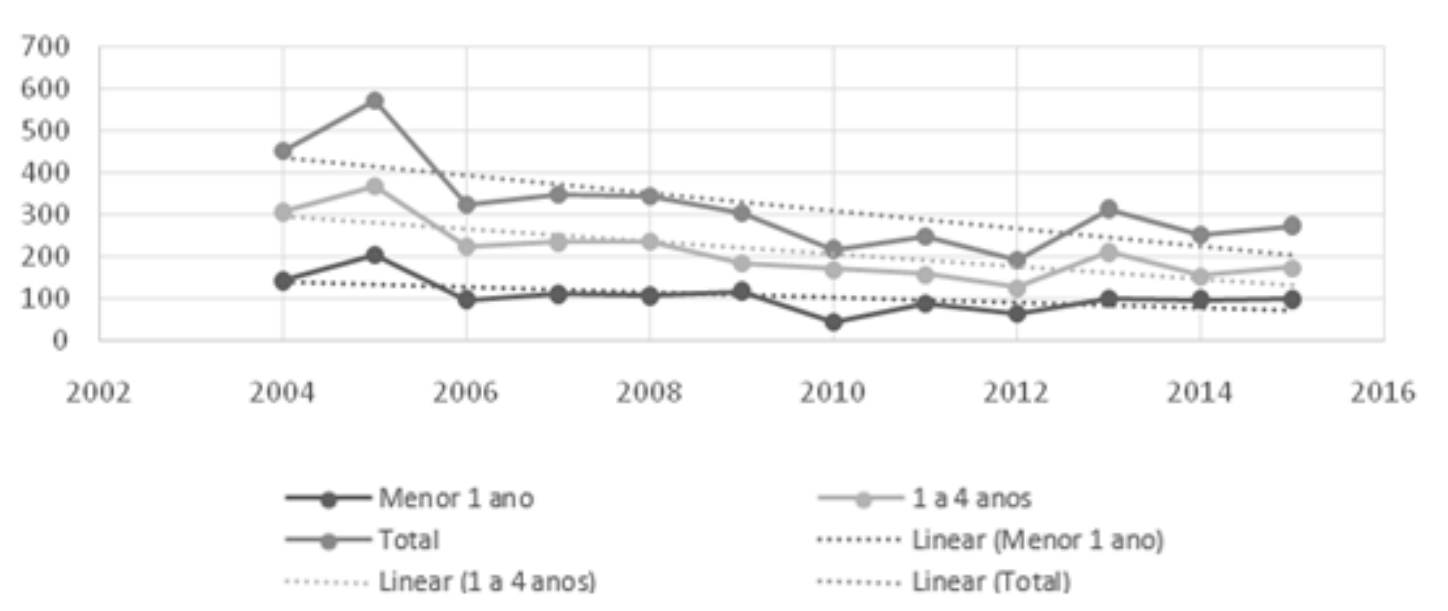

Figura 2. Número de Internações de crianças com pneumonia grupo etário no período pré vacinal e pós vacinal, RIDE-Gurupi-TO $\left(y=-21,14 x+42815 ; R^{2}=0,529\right)$. 


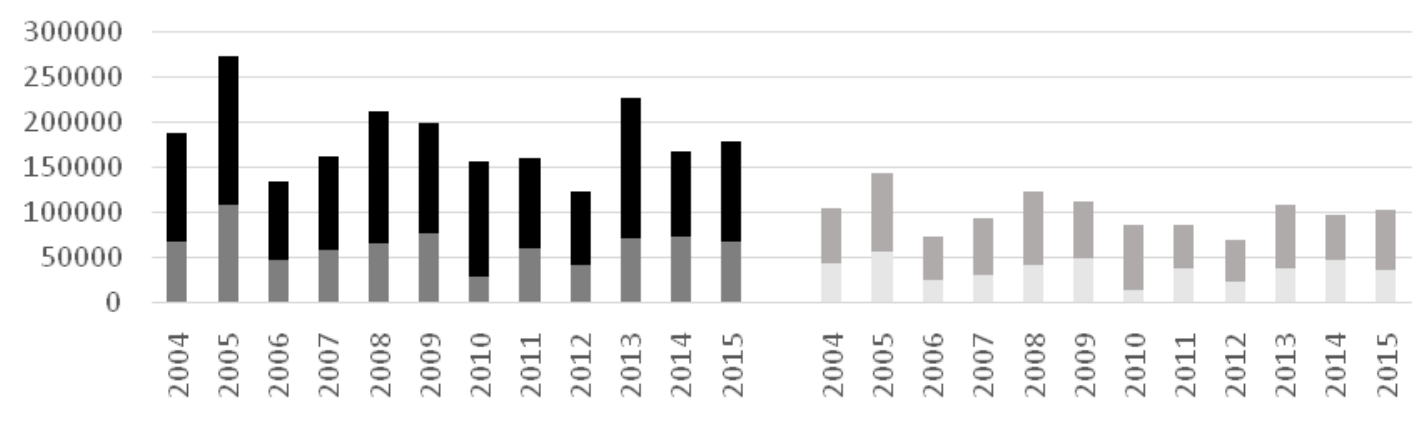

- Masculino menor de 1 ano

Masculino de 1 a 4 anos

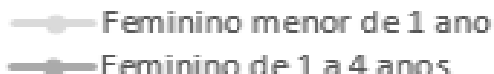

Figura 3. Gastos hospitalares no período pré vacinal e pós vacinal com PCV10 em crianças por grupo etário e de gênero, RIDE-Gurupi-TO.

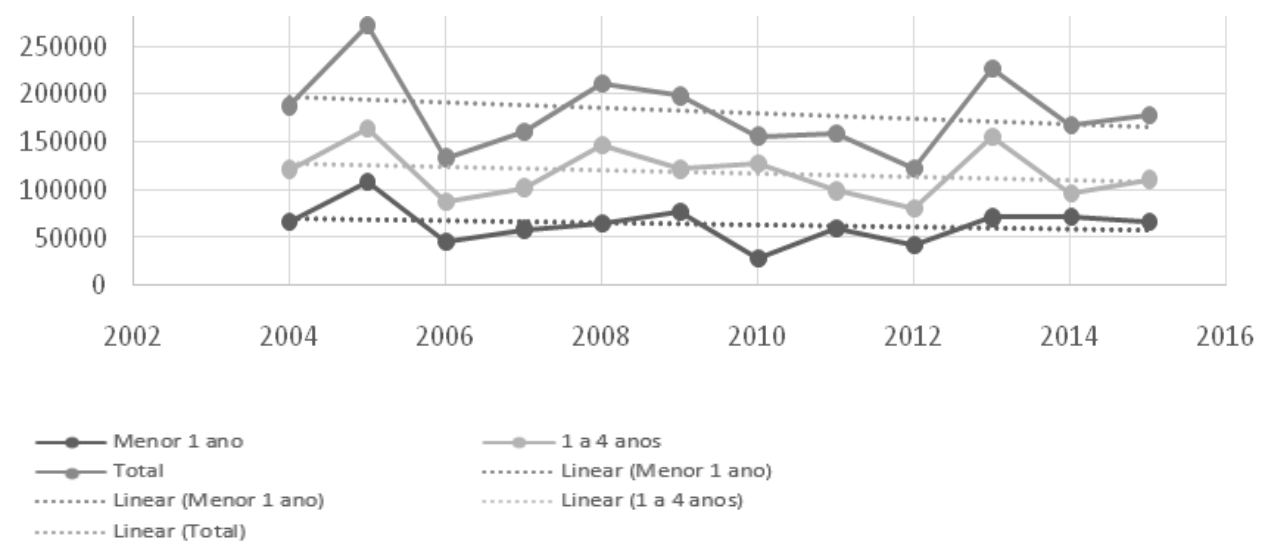

Figura 4. Gastos hospitalares no período pré vacinal e pós vacinal com PCV10 por grupo etário e de gênero, RIDE-Gurupi-TO ( $\left.y=-2757,7 x+6 E+06, R^{2}=0,0572\right)$.

Para análise de correlação foram utilizadas as variáveis cobertura vacinal, número de internações e gastos com internações associando todos os grupos $(A+B)$ e $(1+2)$ ao longo dos anos (Tabela 1 ).

Nasceram 250.996 crianças em Tocantins, com média anual de 25.099,6, sendo nascidos e média anual na RIDE-Gurupi, 28.845 e 2.884,5, respectivamente, no período de 2005 a 2014. Quando analisados os números de nascidos vivos ao longo dos anos verifica-se em Tocantins e em Gurupi as seguintes relações, respectivamente: $\mathrm{y}=-192,27 \mathrm{x}+$ 26.157; $R^{2}=0,6391 ; y=-49,8 x+$ 102.958; $R^{2}=0,7485$ (Figura 5).

Quanto a cobertura vacinal por PCV10 foi de $88,9 \%$ em grupos de imunização no período de 2004 a 2015 sendo: em 2010 (43,39\%), 2011 (102,08\%), 2012 (97,1\%), 2013 $(96,27 \%), 2014 \quad(99,27 \%)$ e 2015 $(95,3 \%)$. Foram aproximadamente 
63.652 doses subdivididas: em $1^{\circ}, 2^{\circ}$, $3^{\circ}$ e dose reforço, representado na figura 6.

Observa-se que houve redução do número de internações por pneumonia com a imunização de crianças na RIDE-Gurupi-TO, considerando grupos etários e de gênero antes e depois da cobertura vacinal (Tabela 1) bem como também foram reduzidos os gastos em saúde no período analisado (Tabela 2).

Para os nascidos vivos no estado de Tocantins e RIDE - Gurupi, observase que também houve redução (Tabela $3)$.

De maneira geral tem-se que ao analisarmos as internações RIDE
Gurupi- TO verificamos a maior incidência no Grupo B com 66,77\% dos casos, com sutil predomínio de 55,09\% do grupo masculino. Quando avaliamos o período pré-vacinal, em relação ao pós-vacinal, no Grupo $A+B$ houve redução $(-36 \%)$ do número de internações $(p=0,011)$. Em relação às faixas etárias o grupo A (-37,17\%; $\mathrm{p}<0,05)$ e 0 grupo B $(-35,91 \%$; $\mathrm{p}=0,0096)$ tiveram reduções significantes. Em relação ao gênero, o grupo $\mathrm{A} 1+\mathrm{B} 1 \quad(-38,37 \% ; \quad \mathrm{p}=0,0038)$, grupo A1 (-37,17\%; $p=0,015)$ e B1 ($49 \% ; \quad p=0,0047$ ) apresentou uma redução maior e mais significativa que o grupo feminino A2+B2 (-32,92\%; $p=0,013), A 2(-34,76 \% ; p=0,047), B 2(-$ $32,15 \% ; p=0,032)$.

Tabela 1. Correlações entre cobertura vacinal PCV10, número de internações e gastos hospitalares em crianças por pneumonia na RIDE de Gurupi-TO, período de 2004 a 2015.

\begin{tabular}{cccc}
\hline & Cobertura vacinal & $\begin{array}{c}\text { Número de } \\
\text { internações }\end{array}$ & $\begin{array}{c}\text { Gastos com } \\
\text { internações }\end{array}$ \\
\hline $\begin{array}{c}\text { Cobertura } \\
\text { vacinal }\end{array}$ & 1 & & \\
$\begin{array}{c}\text { Número de } \\
\text { internações }\end{array}$ & -0.63749 & & \\
$\begin{array}{c}\text { Gastos } \\
\text { com }\end{array}$ & $-0.0258)$ & 1 & 1 \\
internações & $(0.3738)$ & 0.74806 & \\
\hline
\end{tabular}




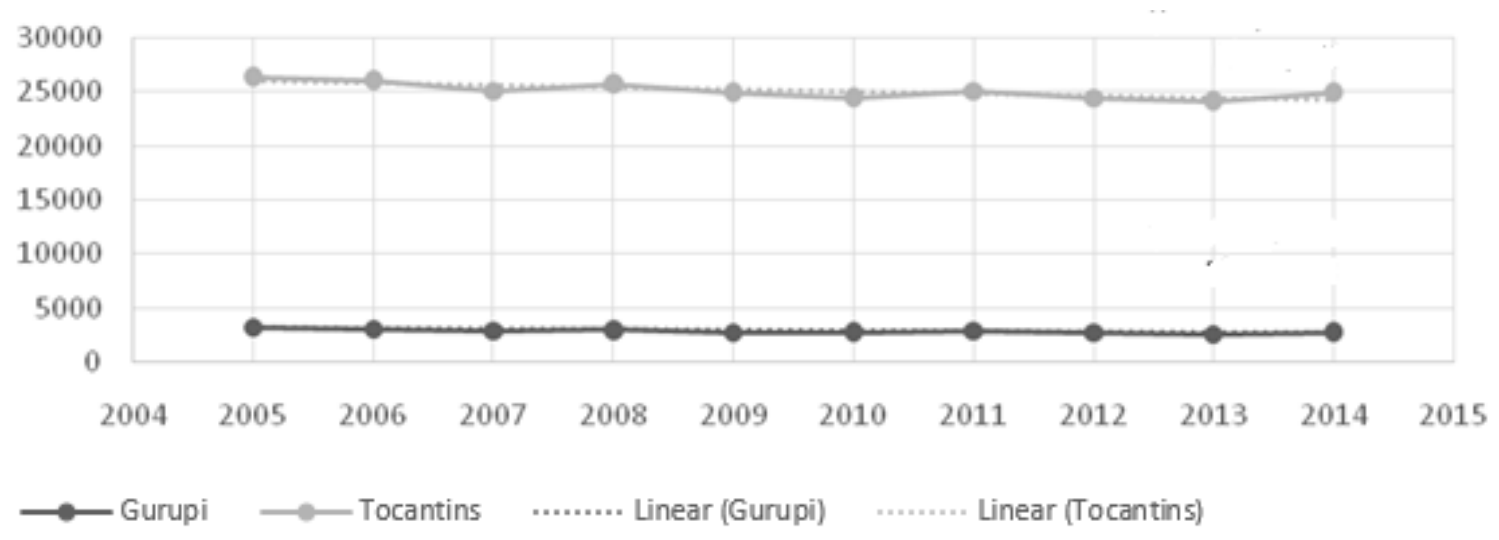

Figura 5. Número de nascidos vivos no Estado do Tocantins e no município de Gurupi no período de 2004 a $2014\left(y=-192,27 x+26.157 ; R^{2}=0,6391 ; y=-49,8 x+\right.$ 102.958; $\left.R^{2}=0,7485\right)$.

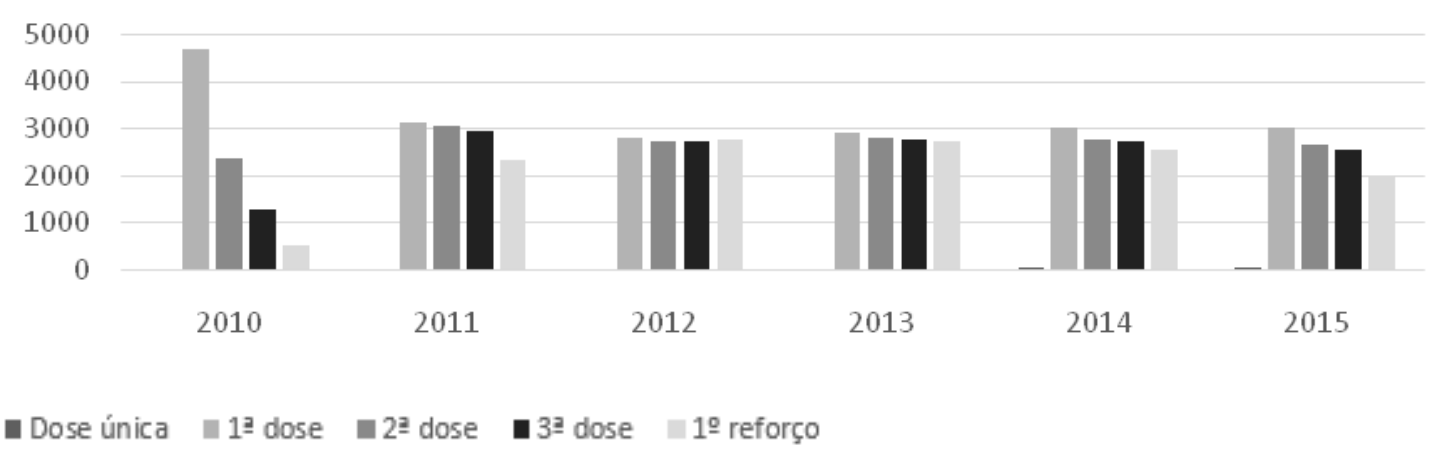

Figura 6. Cobertura vacinal por PCV10 em grupos de imunização de crianças no período de 2004 a 2015, RIDE-Gurupi-TO.

Tabela 2. Análise comparativa entre o número de internações de crianças com pneumonia na RIDE-Gurupi-TO e grupos etários e de gênero antes e depois da cobertura vacinal de PCV10.

\begin{tabular}{llllc}
\hline Grupos & Antes (no) & Depois $\left(\mathbf{n}^{\circ}\right)$ & Redução (\%) & $\mathbf{P}$ \\
\hline A + B & 2.345 & 1.501 & $-36,00$ & $\mathrm{P}=0,011$ \\
A & 780 & 498 & $-37,17$ & $\mathrm{P}<0,05$ \\
B & 1.565 & 1.003 & $-35,91$ & $\mathrm{P}=0,009$ \\
A1 + B1 & 1.311 & 808 & $-38,37$ & $\mathrm{P}=0,003$ \\
A1 & 452 & 284 & $-37,17$ & $\mathrm{P}=0,015$ \\
B1 & 859 & 524 & $-49,00$ & $\mathrm{P}=0,004$ \\
A2 + B2 & 1.034 & 693 & $-32,98$ & $\mathrm{P}=0,013$ \\
A2 & 328 & 214 & $-34,76$ & $\mathrm{P}=0,047$ \\
B2 & 706 & 479 & $-32,15$ & $\mathrm{P}=0,032$ \\
\hline
\end{tabular}


Tabela 3. Análise comparativa dos gastos totais com internações de crianças por pneumonia por grupos etários e de gênero antes e depois da cobertura vacinal PCV-10 na RIDE-Gurupi-TO.

\begin{tabular}{llllc}
\hline Grupos & Antes (no) & Depois (nº) & Redução (\%) & P \\
\hline A + B & $1.164 .909,36$ & $1.010 .993,76$ & $-13,21$ & $P=0.306$ \\
A & $422.358,34$ & $341.852,21$ & $-19,1$ & $P=0.262$ \\
B & $742.551,02$ & $669.141,55$ & $-9,89$ & $\mathrm{P}=0.456$ \\
A1 + B1 & $648.627,45$ & $549.548,37$ & $-15,28$ & $\mathrm{P}=0.180$ \\
A1 & $244.520,04$ & $197.799,56$ & $-19,11$ & $\mathrm{P}=0.279$ \\
B1 & $404.107,41$ & $351.748,81$ & $-12,96$ & $\mathrm{P}=0.286$ \\
A2 + B2 & $516.281,91$ & $461.445,39$ & $-10,62$ & $\mathrm{P}=0.037$ \\
A2 & $177.838,3$ & $144.052,65$ & -19 & $\mathrm{P}=0.242$ \\
B2 & $338.443,61$ & $317.392,74$ & $-6,22$ & $\mathrm{P}=0.712$ \\
\hline
\end{tabular}

Tabela 4. Nascidos vivos antes e após o período de cobertura vacinal com a PCV-10 na RIDE Gurupi e Estado do Tocantins.

\begin{tabular}{llllc}
\hline Local & Antes (n) & Depois (n) & Redução (\%) & P \\
\hline RIDE - Gurupi & 15022 & 13823 & $-7,981$ & $\mathrm{P}=0,028$ \\
Tocantins & 128031 & 122965 & $-3,956$ & $\mathrm{P}=0,015$ \\
\hline
\end{tabular}

\section{DISCUSSÃO}

A introdução da vacina PCV10 no Estado do Tocantins e RIDE Gurupi, por meio do Programa de Imunização Brasileiro a partir de 2010, resultou numa redução da taxa de internações por pneumonia em crianças. A análise do grupo de nascidos vivos demonstrou redução significativa da taxa de nascimento, sem influência significativa sob as internações por pneumonia na RIDE Gurupi. Evidenciou-se também redução dos gastos com internações por pneumonia, sendo significativo apenas no grupo geral feminino. Constata-se que as taxas de hospitalização por pneumonia e outras doenças não-respiratórias causadas pelo pneumococo foram reduzidas no período pós-vacinal.

Este resultado foi similar a estudos anteriores. Afonso et al. (2013), ao analisar o efeito da PCV10 na doença pneumocócica em crianças menores de 2 anos, verificaram 
redução da taxa de hospitalização em Belo Horizonte $(-40,3 \%)$, Curitiba ($37,6 \%)$ e Recife (-49,3\%). Grando et al. (2015) verificaram redução do número de casos de incidência de meningite pneumocócica nas crianças menores de dois anos, em 2012. Abrão et al. (2015) relatam que após análise de registros de avaliação e visitas ambulatoriais durante o período pós introdução da PCV10 foi verificada uma redução significativa na prevalência de Pneumonia Adquirida na Comunidade (PAC), o que sugere a eficácia da vacina. Na cidade de Guaranésia-MG a análise realizada por Abrão et al. (2015) envolvendo grupos etários de 12 meses para o sexo masculino e de 13 meses para o feminino verificaram também redução significativa $(-40 \%)$ nos casos PAC por S. pneumoniae após a introdução da PCV10. Madhi (2014) cita um estudo no Paraná em que houve uma redução de $60 \%$ nos casos de meningite pneumocócica pós PCV10.

Em estudo de caso-controle envolvendo crianças chilenas nascidasvivas nos anos de 2010 e 2011 foi estimado que a PCV10 apresenta um efeito protetor no número de hospitalizações e mortes por pneumonia (inclusive viral) e, quando especificado o grupo etiológico (S. pneumoniae), verifica-se um acréscimo moderado da eficácia da vacina (DIAZ et al, 2016). Em Santa Catarina em estudo ecológico foi verificado uma redução não significativa do número de mortes no período pós-vacinal. (KUPEK e VIEIRA, 2016). No estado de Goiás em recente estudo avaliando a hospitalização por gênero foi verificada uma maior tendência de hospitalização de meninos, entretanto, na análise pósvacina observou redução considerável de meninas decorrentes da doença, quando comparados com meninos (SGAMBATTI, 2015).

Ao comparar os grupos de nascidos vivos, em relação às internações do período pré e pós vacinal da RIDE de Gurupi- TO, houve redução $(-7,98 \% ; p=0,028)$ assim como no Estado do Tocantins (-3,96\%) $(p=0,015)$. Ao comparar o número de internação por pneumonia em crianças de ambos os sexos e idade entre 0 e 4 anos na RIDE de Gurupi-TO, em períodos pré e pós vacinal, considerando os 12 anos analisados, verificou-se que o modelo evidenciou um coeficiente de determinação $\left(r^{2}=\right.$ 0,5293 ) moderado enquanto para nascidos vivos em relação ao período analisado o coeficiente $\left(r^{2}=0,7485\right)$ foi 
forte, ficando mais próximo da linha de regressão ajustada. A correlação entre os dados analisados ao longo dos anos, cujas variáveis foram a cobertura vacinal e número de internações, foi negativa moderada $(p=0.0258)$, onde $o$ número de internações diminui após presença da PCV10 após 2010. Já para análise quanto aos gastos e número de internações no período analisado verificou-se a correlação positiva forte $(p=0.0051)$, demonstrando efetividade da cobertura vacinal a partir da redução de gastos com internações por pneumonia em crianças de 0 a 4 anos. Tem-se, portanto, uma análise a partir de nascidos vivos nesses 12 anos mostrando que a diferença da redução de nascimentos foi bem menor que a diferença de internações ocorridas entre o período pré e pós vacinal, evidenciando a efetividade da PCV10 em relação a redução hospitalar de pneumonia em crianças. Este é um parâmetro útil neste tipo de avaliação, mas não deve ser analisado isoladamente para explicar a redução do número de internações após a imunização.

Kupek e Vieira (2016) ao avaliarem o impacto da taxa de mortalidade, nascidos vivos e presença da PCV10 verificaram uma relação direta sob a taxa de mortalidade apenas em cidades de pequeno porte, com menos de 30 nascidos vivos por ano e, quando avaliaram cidades com portes maiores e mais nascidos vivos a correlação com a PCV10 foi bem menor, o que sugere uma avaliação da qualidade da atenção básica em grandes centros.

Em relação aos gastos hospitalares por valores totais $(R \$)$ houve uma redução nos grupos $A+B$ ($13,21 \%)(p=0,3), A(-19,1 \%)(p=0,26)$, B $(-9,89 \%)(p=0,45) ; A 1+B 1 \quad(-15,28)$ $(p=0,18), A 1(-19,11 \%)(P=0,27), B 1 \quad-$ $12,96 \%) \quad(p=0,28) ; \quad A 2+B 2 \quad(-10,62 \%)$ $(p=0,037)$, A2 $(-19 \%)(p=0,24), B 2(-$ $6,22 \%) \quad(p=0,71)$. Entretanto, houve diferença significativa apenas na redução por grupo geral feminino entre períodos, embora reduzidos também para o sexo masculino e grupo etário.

De acordo com Dal Moro et al. (2015) tem-se uma média de gasto de 905 reais por internação em crianças com pneumonia, sendo total de $\mathrm{R} \$ 1.026 .549,68$ reais representando $28 \%$ das doenças respiratórias. No presente estudo constata-se que 0 gasto da RIDE-Gurupi foi bem menor, com média de $R \$ 565,75$ reais por internação de pneumonia. Segundo dados produzidos pelo CCATES 
(2015), a estimativa de gastos no país por ano é de $\mathrm{R} \$ \mathbf{5 6 0 . 9 4 6 . 7 8 0 , 0 0}$ milhões de reais para imunização, em relação aos 2.909.475 nascidos vivos que se enquadram no calendário vacinal, com esquema vacinal (192,8 reais, cada), o que coloca a região RIDE de Gurupi com gasto vacinal até o momento de aproximadamente $\mathrm{R} \$$ 12.272.105,6 milhões de reais. Este custo é, a princípio, elevado se analisado apenas na perspectiva abordada no presente estudo, o que sugere uma avaliação mais criteriosa nesta questão visando direcionar ações eficientes para otimizar os resultados positivos da cobertura vacinal com a PCV10. Em Guarulhos-SP, após um estudo com crianças de zero a 4 anos, de 2008 a 2012, observou que doenças pulmonares estão entre maiores gastos na saúde de acordo com a faixa etária infantil, demonstrando a importância de uma eficácia na atenção básica como indutora da redução dos gastos (MELO et al., s/d).

Importante lembrar que essas análises são feitas em grupos que obtiveram cobertura vacinal de $88 \%$ das crianças na região estudada, e que os grupos de internação e gastos por valores totais não relacionam os gastos dos grupos de imunizações. Considerando que o primeiro ano na RIDE de Gurupi obter apenas 43,39\% de cobertura, não foi diferente em outros estudos, como realizado por Saraiva (2014) no município de Goiânia, que teve apenas $53,4 \%$ das crianças imunizadas no primeiro ano.

Estudos dessa natureza são importantes em medida que auxilia na caracterização do perfil epidemiológico de crianças vacinadas com PCV-10 em escala regional e/ou local, contribuindo para avaliações dessa política pública na perspectiva da cobertura vacinal e viabilidade econômica da medida preventiva. Dentre os benefícios tem-se minimização das internações hospitalares e consequente custos de internações, além da contribuição na redução de morbidade e mortalidade da população. Como limitação do presente estudo, por utilizar a base dados do DATASUS e internações do sistema público de saúde e conveniada com o SUS, tem-se a não totalidade da representação, visto que não contempla informações decorrentes da rede hospitalar privada.

\section{CONSIDERAÇÕES FINAIS}




\begin{abstract}
Os gastos públicos com internações por pneumonia em crianças foram, de maneira geral, menores a partir da imunização com a PCV10 na RIDE de Gurupi-TO, embora com significância estatística apenas na totalidade do grupo feminino. Houve uma redução significativa no número de
\end{abstract}

internação no período pós-vacinal, independentemente do grupo etário e gênero. Apesar de ter diminuído o número de nascidos vivos na RIDEGurupi-TO não se pode atribuir apenas a este fato isolado a redução do número de internação após a imunização.

\section{REFERÊNCIAS}

AFONSO, Eliane Terezinha et al. Effect of 10 -valent pneumococcal vaccine on pneumonia among children, Brazil. Emerg Infect Dis, v. 19, n. 4, p. 589-97, 2013.

ABRÃO, Wanderci Marys Oliveira et al. Impact of the antipneumococcal conjugate vaccine on the occurrence of infectious respiratory diseases and hospitalization rates in children. Revista da Sociedade Brasileira de Medicina Tropical, v. 48, n. 1, p. 44-49, 2015.

AZEVEdO, Luciano Cesar Pontes; TOSCANO, Cristiana M.; BIERRENBACH, Ana Luiza. Bacterial meningitis in Brazil: baseline epidemiologic assessment of the decade prior to the introduction of pneumococcal and meningococcal vaccines. PLoS One, v. 8, n. 6, p. e64524, 2013.

BRASIL. Ministério da Saúde. Banco de dados do Sistema Único de Saúde DATASUS. Disponível em: <http://www2.datasus.gov.br/DATASUS/index.php>, acessado em 25 de fevereiro de 2016.

BRASIL. Ministério da Saúde. Resumo executivo: Saúde Brasil 2012: uma análise da situação de saúde e dos $\mathbf{4 0}$ anos do Programa Nacional de Imunizações [recurso eletrônico]/Ministério da Saúde, Secretaria de Vigilância em Saúde, 
Departamento de Análise de Situação em Saúde. - Brasília: Ministério da Saúde, 2014. p.18:39. Disponível em: www.saude.gov.br/bvs. Acesso em: 25 de fevereiro de 2016.

CCATES. Eficácia, segurança e custo-efetividade da vacina pneumo-13-valente. Centro Colaborador Do Sus: Avaliação de Tecnologias e Excelência em Saúde. CCATES (2015). (Parecer Técnico-Científico). $<$ http://www.ccates.org.br/content/ pdf/PUB 1438180763.pdf>.

DIAZ, Janepsy et al. Effectiveness of the 10-Valent Pneumococcal Conjugate Vaccine (PCV-10) in Children in Chile: A Nested Case-Control Study Using Nationwide Pneumonia Morbidity and Mortality Surveillance Data. PloS one, v. 11, n. 4, p. e0153141, 2016.

DAL MORO, Victória Carneiro et al. Hospitalização e Mortalidade por Pneumonia Infantil em Montes Claros e Minas Gerais entre 2008 e 2012. Renome, v. 4, n. 2, p. 19-28, 2016.

GOMES, Lucy et al. Fatores de risco e medidas profiláticas nas pneumonias adquiridas na comunidade. J Pneumol, v. 27, n. 2, p. 97-114, 2001.

GRANDO, Indianara Maria et al. Impacto da vacina pneumocócica conjugada 10valente na meningite pneumocócica em crianças com até dois anos de idade no Brasil. Cad Saúde Pública, v. 31, n. 2, p. 1-9, 2015. [Internet]. 2015 Feb [cited 2016 May 01]; 31(2): 276-284.

KUPEK, Emil; VIEIRA, Ilse Lisiane Viertel. O impacto da vacina pneumocócica 5 na redução da mortalidade por pneumonia em crianças menores de um ano en. Catarina, Brasil. Cadernos de Saúde Pública, v. 32, n. 3, 2016.

MADHI, Shabir A. Pneumococcal conjugate vaccine and changing epidemiology of childhood bacterial meningitis. Jornal de pediatria, v. 91, n. 2, p. 108-110, 2015. 
MELO, Maykon Diego et al. Gastos com internações por condições sensíveis à atenção primária em crianças de 0 a 4 anos: uma análise descritiva. Associação Brasileira de Economia da Saúde (Resumos). p.9. s/d. http://abresbrasil.org.br/sites/default/files/avaliacao_trabalho_09_gastos_com_intern acoes_por_condicoes_sensiveis_a_atencao_primaria_em_criancas_de_0_a_4_anos _uma_analise_descr.pdf.

MENEZES, Ana Paula de O. et al. Serotype distribution and antimicrobial resistance of Streptococcus pneumoniae prior to introduction of the 10-valent pneumococcal conjugate vaccine in Brazil, 2000-2007. Vaccine, v. 29, n. 6, p. 1139-1144, 2011.

SARAIVA, Fabricia Oliveira et al. Cobertura vacinal e adesão aos esquemas recomendados da vacina pneumocócica conjugada 10 valente logo após sua introdução no calendário básico em Goiânia, GO: estudo transversal. 2014. 98p. (Dissertação de Mestrado UFG, Instituto de Patologia Tropical e Saúde Pública).

YOSHIOKA, Cristina RM et al. Analysis of invasive pneumonia-causing strains of Streptococcus pneumoniae: serotypes and antimicrobial susceptibility. Jornal de pediatria, v. 87, n. 1, p. 70-75, 2011.

TOCANTINS.

do estado. http://central3.to.gov.br/arquivo/241593/http://central3.to.gov.br/arquivo/241593/ Data de acesso: 08/04/2016.

Recebido em: 18/06/2016

Aprovado em: 10/04/2017 